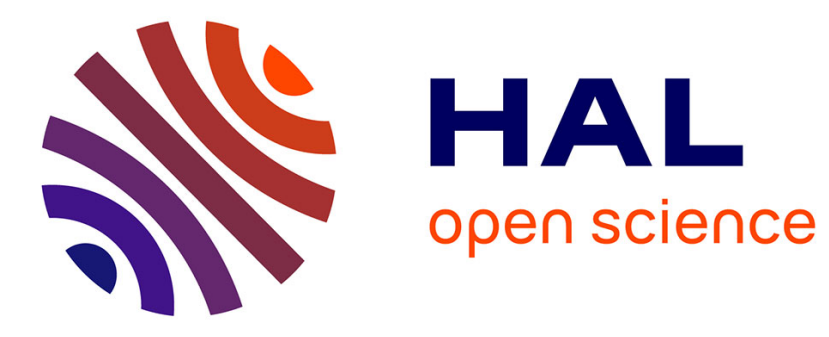

\title{
New Criteria for Blind Equalization Based on PDF Fitting
}

Souhaila Fki, Malek Messai, Abdeldjalil Aissa El Bey, Thierry Chonavel

\section{To cite this version:}

Souhaila Fki, Malek Messai, Abdeldjalil Aissa El Bey, Thierry Chonavel. New Criteria for Blind Equalization Based on PDF Fitting. SPAWC 2014: 15th IEEE International Workshop on Signal Processing Advances in Wireless Communications, Jun 2014, Toronto, Canada. pp.489 - 493. hal01057136

\section{HAL Id: hal-01057136 \\ https://hal.science/hal-01057136}

Submitted on 21 Aug 2014

HAL is a multi-disciplinary open access archive for the deposit and dissemination of scientific research documents, whether they are published or not. The documents may come from teaching and research institutions in France or abroad, or from public or private research centers.
L'archive ouverte pluridisciplinaire HAL, est destinée au dépôt et à la diffusion de documents scientifiques de niveau recherche, publiés ou non, émanant des établissements d'enseignement et de recherche français ou étrangers, des laboratoires publics ou privés. 


\title{
New Criteria for Blind Equalization Based on PDF Fitting
}

\author{
Souhaila Fki, Malek Messai, Abdeldjalil Aïssa-El-Bey, and Thierry Chonavel \\ Institut Mines - Télécom; Télécom Bretagne, Université européenne de Bretagne, UMR CNRS 6285 Lab-STICC \\ Technople Brest Iroise CS 8381829238 Brest, France \\ Email: Firstname.Lastname@telecom-bretagne.eu
}

\begin{abstract}
In this paper, we address M-QAM blind equalization based on information theoretic criteria. We propose two new cost functions that force the probability density functions (pdf) at the equalizer output to match the known constellation pdf. They involve kernel pdf approximation. The kernel bandwidth of a Parzen estimator is updated during iterations to improve the convergence speed and to decrease the residual error of the algorithms. Unlike related existing techniques, the new algorithms measure the distance error between observed and assumed pdfs for the real and imaginary parts of the equalizer output separately. We show performance and complexity gain against the CMA, the most popular blind equalization technique, and classical pdf fitting approaches.
\end{abstract}

\section{INTRODUCTION}

In transmissions, multipath propagation introduces intersymbols interference (ISI) that can make it difficult to recover transmitted data. Thus, an equalizer can be used to reduce the ISI. Blind equalization has been an intensive research area for several decades. It aims at developping effective and low complexity algorithms that avoid bandwidth waste resulting from training data. There exist many blind algorithms. The Godard algorithm [1] and the Constant Modulus Algorithm (CMA) [2] which is a particular case of Godard algorithm, are probably the most popular blind equalization techniques. However, they require a long data sequence to converge and show relatively high residual error. To overcome these limitations, several approaches have been proposed in the literature. For instance, we can mention The Multi-Modulus Algorithm (MMA) that performs blind equalization and carrier phase recovery simultaneously [3]. The $\min \ell_{1}$-MMA and MGaussMMA algorithms [4] outperform the MMA by combining the multi-modulus criterion with an alphabet-matching penalty term.

In the last decade, new techniques for blind equalization, based on information theoretic criteria and pdf estimation of transmitted data, have been proposed. These criteria are optimized adaptively, in general by means of stochastic gradient techniques. Among these techniques, the Kullback-Leibler Divergence (KLD) between the pdf at the equalizer output and the known constellation pdf has been proposed in [5]. The Euclidean distance has also been proposed in [6]. It uses Parzen window with Gaussian kernels for pdf estimation. In [7], a technique based on fitting the pdf of the equalizer output at some relevant points that are determined by the modulus of the constellation symbols was proposed. It is known as sampled-pdf fitting. The authors of [7] also proposed in [8] the stochastic blind equalization approach that uses the quadratic distance (SQD) between the pdf at the equalizer output and the known constellation pdf as a cost function. Many digital transmission systems with a high number of states use QAM modulations. As the multi-modulus approaches are well suited for such modulations, we propose to use these techniques to equalize QAM constellations. Therefore, in this paper, we propose a new family of blind algorithms based on the SQD fitting, that we call Multi-Modulus SQD- $\ell$ p (MSQD- $\ell p)$. Unlike the method in [8], MSQD- $\ell p$ measures the distance error between observed and assumed pdfs for real and imaginary parts of the equalizer output separately. The advantage of proceeding this way is that involved distributions show less modes, leading thus to reduced complexity, while preserving phase recovery as for multi-modulus methods. These techniques are designed for multilevel modulations, work at the symbol rate and admit a simple stochastic gradient-based implementation. For pdf estimation, we use the Parzen window with Gaussian kernels. The proposed methods outperform CMA and classical pdf fitting approaches, in terms of convergence speed, residual error and complexity.

This paper is organized as follows. In section II, we present the blind equalization problem and the SQD pdf fitting method. In section III, we propose the new cost functions and their corresponding stochastic gradient expressions. Simulation results and computational complexity are presented in section IV. Conclusions of our work are given in section $\mathrm{V}$.

\section{SIGNAL AND EQUALIZER MODEL}

\section{A. Signal model}

The baseband model of a transmission system with an adaptive blind channel equalizer is shown in Fig.1, where $s(n), n \in \mathbb{Z}$ is the transmitted symbol at time $n$, that is assumed to be drawn from a QAM constellation. $\boldsymbol{h}=$ $\left[h_{0}, h_{1}, \ldots, h_{L_{h}-1}\right]^{T}$ is the multipath channel finite impulse response (FIR) with order $L_{h}$, while (.) ${ }^{T}$ denotes the transpose operator. $b(n), n \in \mathbb{Z}$ is an additive white Gaussian noise. $x(n), n \in \mathbb{Z}$ is the equalizer input. $\boldsymbol{w}=\left[w_{0}, w_{1}, \ldots, w_{L_{w}-1}\right]^{T}$ is the equalizer impulse response, with length $L_{w} . y(n)$ is the equalized signal at time $n . x(n)$ and $y(n)$ can be modeled as

$$
x(n)=\sum_{i=0}^{L_{h}-1} h_{i} s(n-i)+b(n)
$$

and

$$
y(n)=\sum_{i=0}^{L w-1} w_{i} x(n-i)=\boldsymbol{w}^{T} \boldsymbol{x}(n)
$$


where $\boldsymbol{x}(n)=[x(n), x(n-1), \ldots, x(n-L w+1)]^{T}$.

The

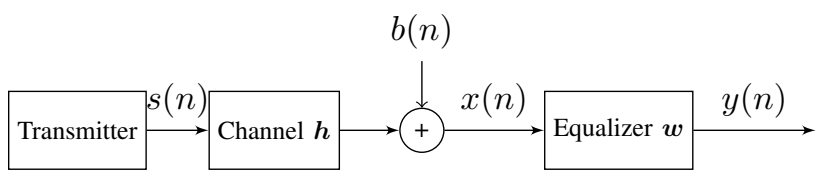

Fig. 1. Baseband model of a transmission system with an adaptive blind channel equalizer.

weights of the equalizer will be adapted by using a gradient stochastic algorithm in the form

$$
\boldsymbol{w}(n+1)=\boldsymbol{w}(n)-\mu \nabla_{\boldsymbol{w}} J(\boldsymbol{w})
$$

where $\mu$ is the step size and $J(\boldsymbol{w})$ is the cost function to be minimized.

\section{B. SQD pdf fitting using Parzen Estimator [8]}

Equalization techniques based on pdf matching intend to minimize some distance between the data distribution at the equalizer output and some target distribution. Transmitted symbols have a discrete distribution. But, since they are affected by additive Gaussian noise at the receiver side, it can be assumed that after removing channel multipath effects, the equalizer output should consist of a Gaussian mixture, with Gaussian modes centered at the constellation points. Therefore, a target distribution of this form can be chosen. Then, the SQD algorithm [8] aims at minimizing the quadratic distance between the pdf of the equalizer output and the pdf of the noisy constellation. Its cost function is given by

$$
J(\boldsymbol{w})=\int_{-\infty}^{\infty}\left(f_{Y^{p}}(z)-f_{S^{P}}(z)\right)^{2} d z
$$

where, $Y^{p}=\left\{|y(n)|^{p}\right\}$ and $S^{p}=\left\{\left|s_{k}\right|^{p}\right\}$ are the sets of the moduli to the power $p$ of equalized symbols and constellation symbols and $f_{Z}(z)$ denotes the pdf of $Z$ at $z$. Thus, $J(\boldsymbol{w})$ is intended to match $p^{t h}$ moment distributions between the equalizer output and the noisy constellation.

By using the Parzen window method with a window involving the $L$ previous symbols, the estimates of the current pdfs are

$$
\begin{array}{r}
\hat{f}_{Y^{p}}(z)=\frac{1}{L} \sum_{k=0}^{L-1} K_{\sigma_{0}}\left(z-|y(n-k)|^{p}\right) \\
\hat{f}_{S^{p}}(z)=\frac{1}{N_{s}} \sum_{k=1}^{N_{s}} K_{\sigma_{0}}\left(z-\left|s_{k}\right|^{p}\right)
\end{array}
$$

where $N_{s}$ is the number of complex symbols in the constellation and $K_{\sigma_{0}}$ is a Gaussian kernel with standard deviation $\sigma_{0}$, also known as the kernel bandwidth:

$$
K_{\sigma_{0}}(x)=\frac{1}{\sqrt{2 \pi} \sigma_{0}} e^{-\frac{x^{2}}{2 \sigma_{0}^{2}}}
$$

According to [8], for $p=2$ and $L=1$, the expression of $J(\boldsymbol{w})$ is given by

$$
\begin{aligned}
J(\boldsymbol{w}) & =\frac{1}{N_{s}^{2}} \sum_{k=1}^{N_{s}} \sum_{l=1}^{N_{s}} K_{\sigma}\left(|s(l)|^{2}-|s(k)|^{2}\right) \\
& -\frac{2}{N_{s}} \sum_{k=1}^{N_{s}} K_{\sigma}\left(|y(n)|^{2}-|s(k)|^{2}\right) .
\end{aligned}
$$

where, $\sigma=\sqrt{2} \sigma_{0}$. Then, the gradient of the cost function with respect to the equalizer weights is given by

$$
\nabla_{\boldsymbol{w}} J(\boldsymbol{w})=-\frac{1}{N_{s}} \sum_{k=1}^{N_{s}} K_{\sigma}^{\prime}\left(|y(n)|^{2}-|s(k)|^{2}\right) y(n) \boldsymbol{x}^{*}(n)
$$

where $K_{\sigma}^{\prime}(x)=-\frac{x}{\sqrt{2 \pi} \sigma^{3}} \exp \left(\frac{-x^{2}}{2 \sigma^{2}}\right)$ is the derivative of $K_{\sigma}(x)$ and $(.)^{*}$ denotes the complex conjugation operator. Then, the equalizer coefficients are updated at symbol rate by inserting Eq.(8) in Eq.(3). This algorithm is initialized with a tapcentered equalizer. In [8], the squared modulus of the symbols for the kernel variables $(p=2)$ is used to design $J(\boldsymbol{w})$. But, squaring does not preserve Gaussianity around noisy constellation points. Then, with a view to make the criterion statistically more meaningful we propose, in this paper, to also address the case $p=1$. Indeed, when $p=1$, since constellation points are apart from the axes, at convergence $|y(n)|$ will be roughly distributed according to a mixture of Gaussian distributions around the constellation points that are in the positive quadrant of the complex plane. This is true provided the SNR remains in usual ranges for QAM modulations under consideration. In addition, it is well known that multimodulus approaches such as MMA [9], that decompose equalization criteria into an in-phase term and a quadrature one, are more efficient than criteria such as the CMA [2], that handle in-phase and quadrature parts together. In the same way, the criteria that we propose are made of a sum of two terms related to in-phase and quadrature parts of the equalizer output. This will lead to criteria that we name Multimodulus SQD- $\ell$ p (MSQD- $\ell$ p). The advantage of proceeding this way is that involved distributions show less modes, leading thus to reduced complexity, while preserving phase recovery. In addition, we benefit from the fact that $1 \mathrm{D}$ pdfs can be accurately estimated with less data than 2D pdfs. Indeed, with the SQD algorithm there are $M$ symbols involved in the target pdf whereas with the MSQD$\ell \mathrm{p}$ algorithms there are only $2 \sqrt{M}$ modes involved, for an M-QAM modulation.

\section{MSQD ALGORITHMS}

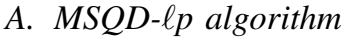

MSQD family consists of algorithms based on cost functions in the form:

$$
\begin{aligned}
J(\boldsymbol{w}) & =\int_{-\infty}^{\infty}\left(\hat{f}_{\left|y_{r}\right|^{p}}(z)-\hat{f}_{\left|s_{r}\right|^{p}}(z)\right)^{2} d z \\
& +\int_{-\infty}^{\infty}\left(\hat{f}_{\left|y_{i}\right|^{p}}(z)-\hat{f}_{\left|s_{i}\right|^{p}}(z)\right)^{2} d z
\end{aligned}
$$

where $y_{r}=\Re\{y\}, y_{i}=\Im\{y\}$ and the pdf estimates are in the form

$$
\hat{f}_{x}(z)=\frac{1}{N_{x}} \sum_{k=1}^{N_{x}} K_{\sigma_{0}}\left(z-x_{k}\right)
$$

$x$ is equal to $\left|s_{r}\right|^{p},\left|s_{i}\right|^{p},\left|y_{r}\right|^{p}$ or $\left|y_{i}\right|^{p} . N_{x}=N_{s}$ for $x=\left|s_{r, i}\right|^{p}$ and $N_{x}=L$ for $x=\left|y_{r, i}\right|^{p}$.

For fixed $p$, we denote the corresponding criterion by MSQD$\ell$ p. In a stochastic gradient optimization approach, in general only instantaneous statistics are involved in the criterion. Thus, 
we consider a window length $L=1$ as in [8]. Then, since for Gaussian kernels we have

$$
\int_{-\infty}^{\infty} K_{\sigma_{0}}\left(y-C_{1}\right) K_{\sigma_{0}}\left(y-C_{2}\right) d y=\frac{1}{2} K_{\sigma_{0} \sqrt{2}}\left(C_{1}-C_{2}\right),
$$

thus, according to Eq.(9) and Eq.(10), $J(\boldsymbol{w})$ becomes

$$
\begin{aligned}
J(\boldsymbol{w}) & =-\frac{1}{N_{s}} \sum_{k=1}^{N_{s}} K_{\sigma}\left(\left|y_{r}(n)\right|^{p}-\left|s_{r}(k)\right|^{p}\right) \\
& -\frac{1}{N_{s}} \sum_{k=1}^{N_{s}} K_{\sigma}\left(\left|y_{i}(n)\right|^{p}-\left|s_{i}(k)\right|^{p}\right)+C s t .
\end{aligned}
$$

Therefore, the derivative of $J(\boldsymbol{w})$ with respect to equalizer weights is

$$
\begin{aligned}
\nabla_{\boldsymbol{w}} J(\boldsymbol{w}) & =\frac{\partial J(\boldsymbol{w})}{\partial \boldsymbol{w}_{r}}+j \frac{\partial J(\boldsymbol{w})}{\partial \boldsymbol{w}_{i}} \\
& =\frac{p}{2 \sqrt{2 \pi} N_{s} \sigma^{3}} \sum_{k=1}^{N_{s}}\left(\operatorname { s i g n } ( y _ { r } ( n ) ) | y _ { r } ( n ) | ^ { p - 1 } \left(\left|y_{r}(n)\right|^{p}\right.\right. \\
& \left.-\left|s_{r}(k)\right|^{p}\right) e^{-\frac{\left(\left|y_{r}(n)\right|^{p}-\left|s_{r}(k)\right|^{p}\right)^{2}}{2 \sigma^{2}}}+j \operatorname{sign}\left(y_{i}(n)\right) \\
& \times\left|y_{i}(n)\right|^{p-1}\left(\left|y_{i}(n)\right|^{p}-\left|s_{i}(k)\right|^{p}\right) \\
& \left.\times e^{-\frac{\left(\left|y_{i}(n)\right|^{p}-\left|s_{i}(k)\right|^{p}\right)^{2}}{2 \sigma^{2}}}\right) \boldsymbol{x}^{*}(n) .
\end{aligned}
$$

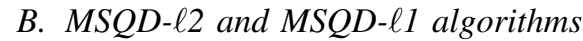

Since $\ell 2$ norm is often considered in the literature and for comparison to the case $p=1$ in the simulation part, we consider first the case $p=2$. From Eq.(13) we get the gradient of the MSQD- $\ell 2$ cost function:

$$
\begin{aligned}
\nabla_{\boldsymbol{w}} J(\boldsymbol{w}) & =\frac{1}{\sqrt{2 \pi} N_{s} \sigma^{3}} \sum_{k=1}^{N_{s}}\left(y_{r}(n)\left(\left|y_{r}(n)\right|^{2}-\left|s_{r}(k)\right|^{2}\right)\right. \\
& \times e^{-\frac{\left(\left|y_{r}(n)\right|^{2}-\left|s_{r}(k)\right|^{2}\right)^{2}}{2 \sigma^{2}}}+j y_{i}(n)\left(\left|y_{i}(n)\right|^{2}-\left|s_{i}(k)\right|^{2}\right) \\
& \left.\times e^{-\frac{\left(\left|y_{i}(n)\right|^{2}-\left|s_{i}(k)\right|^{2}\right)^{2}}{2 \sigma^{2}}}\right) \boldsymbol{x}^{*}(n)
\end{aligned}
$$

Then, Eq.(3) is used to update equalizer taps. For the case $p=1$, that is statistically more meaningful, as discussed at the end of section II, we get an updating term of the equalizer in the form:

$$
\begin{aligned}
\nabla_{\boldsymbol{w}} J(\boldsymbol{w}) & =\frac{1}{\sqrt{8 \pi} N_{s} \sigma^{3}} \sum_{k=1}^{N_{s}}\left(\operatorname{sign}\left(y_{r}(n)\right)\left(\left|y_{r}(n)\right|-\left|s_{r}(k)\right|\right) \times\right. \\
& e^{-\frac{\left(\left|y_{r}(n)\right|-\left|s_{r}(k)\right|\right)^{2}}{2 \sigma^{2}}}+j \operatorname{sign}\left(y_{i}(n)\right)\left(\left|y_{i}(n)\right|-\left|s_{i}(k)\right|\right) \\
& \left.\times e^{-\frac{\left(\left|y_{i}(n)\right|-\left|s_{i}(k)\right|\right)^{2}}{2 \sigma^{2}}}\right) \boldsymbol{x}^{*}(n)
\end{aligned}
$$

In section IV, we will show on simulations that, as expected, the proposed MSQD- $\ell 1$ algorithm is more effective than the existing SQD algorithm in terms of mean square error and convergence speed, especially for larger constellations.

\section{Simulation Results}

\section{A. Adaptive adjustment of the kernel size}

As in [8], the kernel size was adaptively controlled assuming a linear relationship between the kernel size and the decision error:

$$
\sigma(n)=a G(n)+b
$$

where,

$$
G(n)=\alpha G(n-1)+(1-\alpha) \underbrace{\min }_{k=1, \ldots, N_{s}}\left(\left(|y(n)|^{2}-\left|s_{k}\right|^{2}\right)^{2}\right)
$$

$\alpha$ is a forgetting factor and $(a, b)$ are empirically determined constants. As mentioned in [8], the minimum of the stochastic cost function is a scaled version of the desired constellation. Then, the original symbols $\left|s_{k}\right|^{2}$ in Eq.(14) and Eq.(15) are substituted by $\left|s_{k}^{c}\right|^{2}=Q(\sigma)\left|s_{k}\right|^{2}$, where $Q(\sigma)$ is the compensation factor that depends on the kernel size and is obtained by ensuring that the zero-ISI solution $\left(y(n)=s\left(k_{n}\right)\right)$ is a minimum of $\mathbb{E}[J(\boldsymbol{w})]$, that is $\mathbb{E}\left[\nabla_{\boldsymbol{w}} J(\boldsymbol{w})\right]=0$, or

$$
\sum_{k=1}^{N_{s}} \mathbb{E}\left[K_{\sigma}^{\prime}\left(\left|s\left(k_{n}\right)\right|^{2}-Q(\sigma)\left|s_{k}\right|^{2}\right) s\left(k_{n}\right) \boldsymbol{x}^{*}(n)\right]=0
$$

Fig.2 shows the compensation factor $Q(\sigma)$ for 16-QAM, 64QAM and 256-QAM modulations when using the MSQD- $\ell 1$ algorithm. For the MSQD- 11 and MSQD- $\ell 2$, we implement

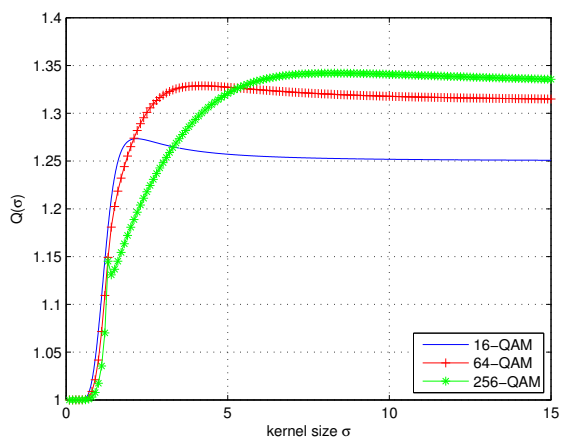

Fig. 2. Evolution of the compensation factor $Q(\sigma)$ for MSQD- $\ell 1$ algorithm.

the same steps as the algorithm summarized in [8], using the appropriate cost functions and $Q$ functions.

\section{B. Numerical results} [8]

In simulations, we first use the channel that was used in

$$
H_{1}=[0.2258,0.5161,0.6452,-0.5161]^{T} .
$$

Performance of the proposed MSQD- $\ell 2$ and MSQD- $\ell 1$ algorithms are compared with those of the CMA, MMA and SQD. For simulations, we employed an equalizer of length $L_{w}=21$ initialized using the tap-centered strategy. Table I summarizes the parameters which were used to draw the curves in Fig.3. To compare the performance of the proposed algorithms in terms of residual error, we set the step size $\mu$ for each algorithm such as they converge with the same speed. Thus, in Fig.3, we can clearly notice that MSQD- $\ell 2$ and MSQD- $\ell 1$ outperform the CMA, MMA and SQD algorithms in terms of residual error 
for 16-QAM and 64-QAM modulations. On the other hand, when we fix the value of $\mu$ for each algorithm such as they converge to the same MSE in Fig.4, we notice that MSQD$\ell 2$ and MSQD- $\ell 1$ converge faster. These figures show that the MSQD- $\ell 1$ converges close to the MSE of the MMSE equalizer. This is in accordance with [10] where we have proved that the MMSE equalizer is the only stationary stable point of the MSQD- $\ell 1$ algorithm. In Fig.5 we show the performance

TABLE I. PARAMETER VALUES USED FOR SIMULATIONS

\begin{tabular}{|c|c|c|c|}
\hline 16 QAM & SQD & MSQD- $\ell 2$ & MSQD- $\ell 1$ \\
\hline$\mu$ & $10^{-4}$ & $1.3 \times 10^{-4}$ & $7.7 \times 10^{-4}$ \\
$a$ & 3.5 & 3.5 & 1.5 \\
$b$ & -9.5 & -9.5 & -1 \\
$1-\alpha$ & $5 \times 10^{-3}$ & $5 \times 10^{-3}$ & $5 \times 10^{-3}$ \\
$E_{0}$ & 7 & 7 & 5 \\
\hline 64 QAM & & & \\
\hline$\mu$ & $1.2 \times 10^{-6}$ & $9 \times 10^{-7}$ & $4.7 \times 10^{-5}$ \\
$a$ & 3.5 & 3 & 2 \\
$b$ & -2 & -18 & -10 \\
$1-\alpha$ & $10^{-3}$ & $10^{-2}$ & $10^{-3}$ \\
$E_{0}$ & 5 & 7 & 6.5 \\
\hline \multicolumn{4}{|r}{} \\
\hline
\end{tabular}

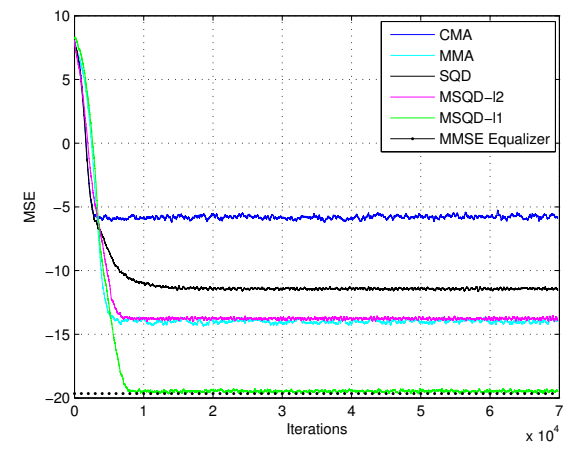

(a) 16-QAM

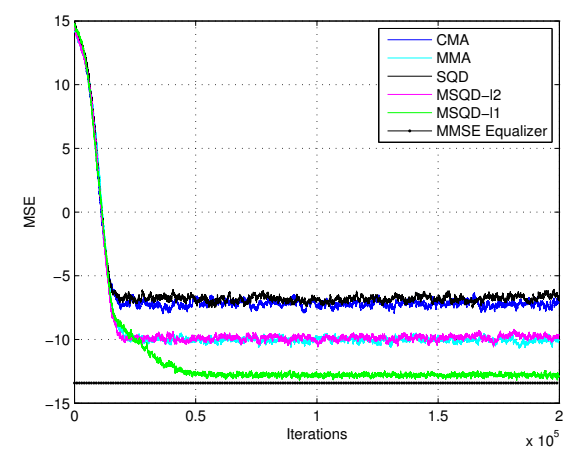

(b) 64-QAM

Fig. 3. MSE $(\mathrm{dB})$ for $\mathrm{SNR}=30 \mathrm{~dB}$ using $H_{1}$.

of the proposed methods when using the channel of length $L_{h 2}=10$ with transfer function $H_{2}(z)=\sum_{l=0}^{L_{h}-1} h_{2}(l) z^{-l}$ with $h_{2}(l) \sim \mathcal{N}\left(0, G e^{-\rho l}\right)$ such that $\sum_{l=0}^{L_{h}-1} \mathbb{E}\left[\left|h_{2}(l)\right|^{2}\right]=1$. For simulations, we chose $\rho=0.7$. We can check that with this channel, the MSQD- $\ell 1$ outperforms the other algorithms and converges to the MMSE equalizer [10].

To study the performance of the MSQD- $\ell 1$ algorithm as a

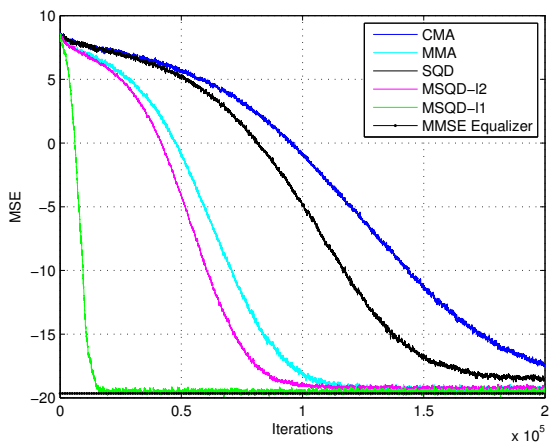

(a) 16-QAM

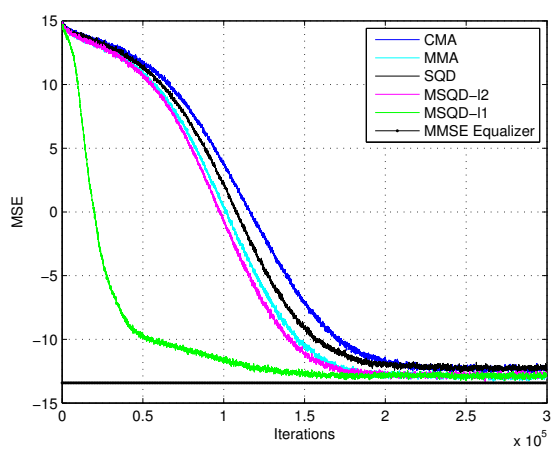

(b) 64-QAM

Fig. 4. MSE (dB) for $\mathrm{SNR}=30 \mathrm{~dB}$ using $H_{1}$.

function of SNR, we draw in Fig.6 the Symbol Error Rate (SER) for the MMA, SQD, MSQD- $\ell 1$ algorithms and for an AWGN channel between $S N R=0 \mathrm{~dB}$ and $\mathrm{SNR}=20 \mathrm{~dB}$ for a 16-QAM modulation. To plot these curves, we take the optimal equalizer for each algorithm with the same convergence rate. It is clear in this figure that the MSQD- $\ell 1$ algorithm outperforms the other algorithms in terms of the SER. We can also notice that for a value of SER equal to $10^{-2}$, the MSQD- $\ell 1$ has a gain of $1.2 \mathrm{~dB}$ compared to the SQD. Moreover, its performance are very close to those obtained with an AWGN channel for any SNR.

\section{Computational complexity analysis}

For a square M-QAM modulation, the computational complexity is summarized in table II where $N_{s}=\frac{(\sqrt{M} / 2) !}{2 !\left(\frac{\sqrt{(M)}}{2}-2\right) !}+$ $\frac{\sqrt{M}}{2}$ and $N_{s}^{\prime}=\frac{\sqrt{M}}{2}$ when $M>4$ and $N_{s}=N_{s}^{\prime}$ when $M^{2}=4$. Therefore, we can conclude that the MSQD- $\ell 1$ is

TABLE II. COMPUTATIONAL COMPLEXITY OF CMA, SQD AND MSQD- $\ell 1$ ALGORITHMS FOR ONE ITERATION

\begin{tabular}{|c|c|c|}
\hline Equalizers & Multiplications & Exponent \\
\hline CMA & $8 L_{w}+4$ & 0 \\
SQD & $4 N_{s}+8 L_{w}+4$ & $N_{s}$ \\
MSQD $\ell 1$ & $6 N_{s}^{\prime}+8 L_{w}+2$ & $2 N_{s}^{\prime}$ \\
\hline
\end{tabular}

computationally less demanding than the SQD and slightly more demanding compared to the CMA. However, it requires many fewer iterations to converge to a low MSE. In Fig.4 we can notice that the MSQD- $\ell 1$ converges about 10 times 


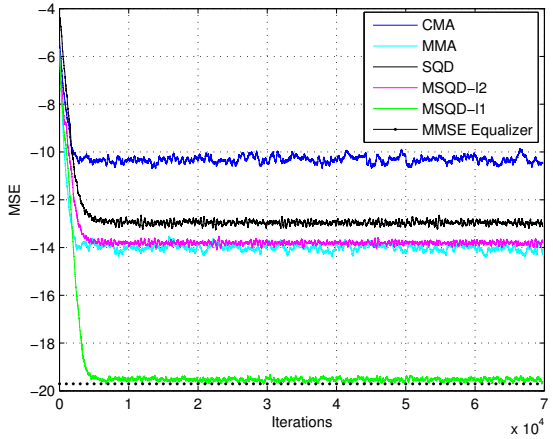

(a) 16-QAM

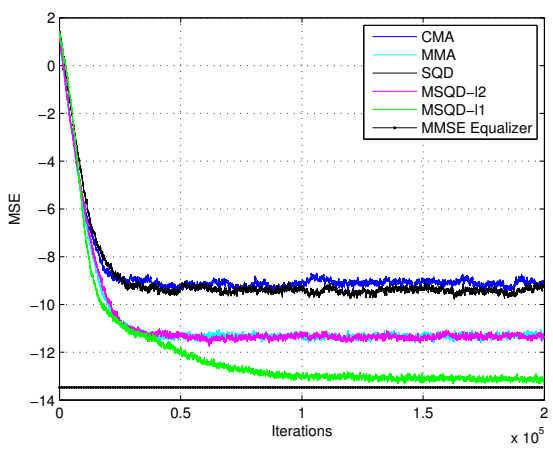

(b) 64-QAM

Fig. 5. MSE (dB) for $\mathrm{SNR}=30 \mathrm{~dB}$ using $\mathrm{H}_{2}$.

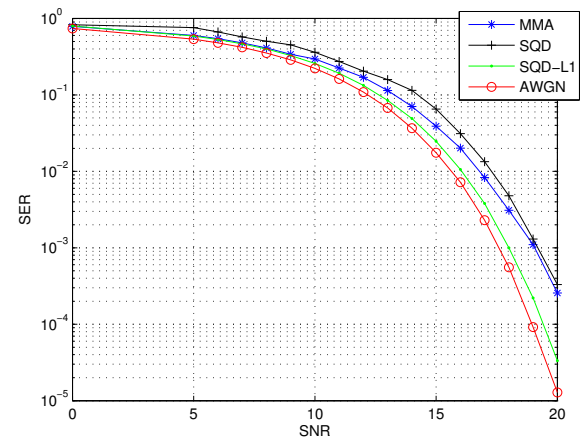

Fig. 6. SER for MMA, SQD, MSQD- $\ell 1$ algorithms using $H_{1}$ and 16-QAM modulation.

faster than the CMA. Fig.7 shows the global computational cost needed to achieve convergence, according to Fig.4. We can notice that the global computational complexity of the MSQD- $\ell 1$, is lower than that of the SQD and the CMA.

\section{CONCLUSION}

In this paper, we have proposed new criteria for kernel based blind equalization techniques that force the pdf of the real and imaginary parts of the equalizer output to match that of the noisy constellation real and imaginary parts by employing the Parzen window method to estimate the data pdf. Performance of the proposed methods has been compared with that of CMA, MMA and SQD. We have shown that they converge faster with a reduced residual error. The behaviour

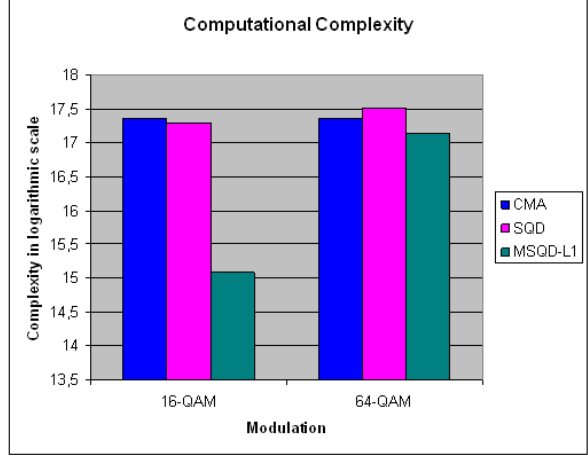

Fig. 7. Number of multiplications needed by the equalizers to converge for $\{16,64\}$-QAM modulations.

of the MSQD- $\ell$, most powerful proposed method, has been examined in [10]. The analysis that we have conducted and simulation results prove that the MSQD- $\ell 1$ algorithm converges to the MMSE equaizer and brings further validation of the pdf fitting approach for equalization in digital transmission. Although in this paper we only addressed QAM modulations, the proposed methods can be extended to any modulation.

\section{REFERENCES}

[1] D. Godard, "Self-recovering equalization and carrier tracking in twodimensional data communication systems," IEEE Transactions on Communications, vol. 28, no. 11, pp. 1867 - 1875, November 1980.

[2] J. Treichler and B. Agee, "A new approach to multipath correction of constant modulus signals," IEEE Transactions on Acoustics, Speech and Signal Processing, vol. 31, no. 2, pp. 459 - 472, April 1983.

[3] K. N. Oh and Y. O. Chin, "Modified constant modulus algorithm: blind equalization and carrier phase recovery algorithm," in IEEE International Conference on Communications, ICC Seattle, vol. 1, June 1995, pp. $498-502$.

[4] A. Labed, T. Chonavel, A. Assa-El-Bey, and A. Belouchrani, "Minnorm based alphabet-matching algorithm for adaptive blind equalisation of high-order qam signals," in European transactions on telecommunications, vol. 24, no. 6, October 2013, pp. 552-556.

[5] J. Sala-Alvarez and G. Vazquez-Grau, "Statistical reference criteria for adaptive signal processing in digital communications," IEEE Transactions on Signal Processing, vol. 45, no. 1, pp. 14 -31, january 1997.

[6] I. Santamaria, C. Pantaleon, L. Vielva, and J. Principe, "Adaptive blind equalization through quadratic pdf matching," Proceedings of the European Signal Processing Conference, Toulouse, France, vol. II, pp. 289-292, September 2002.

[7] M. Lazaro, I. Santamaria, C. Pantaleon, D. Erdogmus, K. E. Hild II, and J. C. Principe, "Blind equalization by sampled pdf fitting," 4th International Symposium on Independent Component Analysis and Blind Equalization, Nara, Japan, pp. 1041-1046, September 2003.

[8] M. Lazaro, I. Santamaria, D. Erdogmus, K. Hild, C. Pantaleon, and J. Principe, "Stochastic blind equalization based on pdf fitting using parzen estimator," IEEE Transactions on Signal Processing, vol. 53, no. 2, pp. 696 - 704, february 2005.

[9] J. Yang, J.-J. Werner, and G. Dumont, "The multimodulus blind equalization and its generalized algorithms," IEEE Journal on Communications, vol. 20, no. 5, pp. 997 -1015, June 2002.

[10] S. Fki, M. Messai, A. Assa-El-Bey, and T. Chonavel, "Blind equalization based on pdf fitting and convergence analysis," Signal Processing, vol. 101, no. 0, pp. 266 - 277, August 2014. 